\title{
Understanding The Mechanism of Mutant HIV-1 Protease Resistance Against Darunavir Using Molecular Dynamic Study
}

\section{Yaser Shabanpour}

Baqiyatallah University of Medical Sciences

\section{Esmaeil Behmard}

Shiraz University of Medical Sciences

Parviz Abdolmaleki

Tarbiat Modares University

Amir Homayoun Keihan ( $\nabla$ ah_keyhan@hotmail.com )

Baqiyatallah University of Medical Sciences

\section{Sharareh Sajjadi}

Islamic Azad University

\section{Research Article}

Keywords: HIV-1 Protease, Darunavir, MD simulation, drug resistance

Posted Date: April 26th, 2021

DOl: https://doi.org/10.21203/rs.3.rs-431285/v1

License: (9) This work is licensed under a Creative Commons Attribution 4.0 International License. Read Full License 


\section{Abstract}

The human immunodeficiency virus type 1 protease (HIV-1 PR) is an important enzyme in life cycle of the HIV virus. It cleaves inactive pre-proteins of the virus and changes them into active proteins. Darunavir suppresses the wild type HIV-1 PR (WT-Pr) activity, but can't inhibit the mutant resistant forms (MUT-Pr). Increasing knowledge about the resistance mechanism can be helpful for designing of more effective inhibitors. In this study, the mechanism of resistance of Ile47val and Ile54Met MUT-Pr strain against Darunavir was investigated. For this purpose, complexes of Darunavir with WT-Pr (WT-Pr-D) and MUT-Pr (MUT-Pr-D) were simulated for $200 \mathrm{~ns}$ and structure, dynamic and energetic properties of both simulations were investigated based on essential dynamics (principal component analysis (PCA)), root mean square fluctuation (RMSF), radial distribution function (RDF), molecular mechanics/Poisson Boltzman surface area (MM/PBSA) energies and etc. Our data revealed that mutations increased the flap tips flexibility and increased the active-site space, probably due to the reduction in hydrophobic forces. So, the protease's conformation changed from closed state to semi-open state. Formation of semi open structure along with a reduction in van der Waals interactions and hydrogen bonds with Darunavir facilitates disjunction of Darunavir from the active-site in MUT-Pr-D.

\section{Introduction}

The human immunodeficiency virus type 1 protease (HIV-1 PR) is an enzyme that cleaves the HIV polyproteins (the Gag and Gag-pol), helping the virus to reach maturity at the last stage of its life cycle ${ }^{1}$. So, the enzyme inhibitors may play an important role in the battle with the acquired immunodeficiency syndrome (AIDS) disease ${ }^{2}$. However, the strains with a mutant protease demonstrate a great resistance against inhibitors ${ }^{3}$. Therefore, understanding the molecular mechanisms underlying the resistance of HIV-1 PR antiviral drugs is crucial for the design of highly potent inhibitors against the drug-resistant strains.

HIV-1 PR is a member of aspartyl protease family, which contains the aspartate residue in its active-site ${ }^{4}$. This protein is a symmetric homodimer that has two identical chains (i.e. A and B), each containing 99 residues ${ }^{5}$. The Asp25, Thr26 and Gly27 residues form the catalytic site in both chains ${ }^{4}$. HIV-1 PR has six main structural segments including fulcrum (A|B:11-22), active-site (A|B:23-30), flap-elbow (A|B:35-42), flap-tip (A|B:43-58), cantilever (A|B:59-75) and interface (A|B:95-99) (Fig. S1) ${ }^{6}$. The flap-tips are glycine-rich domains with hairpin structure, which control the access of substrate/inhibitor to the activesite $^{7}$. It has been shown both computationally and experimentally that the HIV-1 PR has three possible conformations including closed, semi-open and open-like (i.e. curled, open and wide open) conformations that are classified based on the distance between two flap-tips ${ }^{8-12}$. In the ligand-bound form, the flaptips take a downward conformation relative to the active-site (closed state), while the free form permanently takes a semi-open conformation ${ }^{13,14}$. The inhibitor-pressure selected mutations lead to forming open-like conformations and destabilize the closed conformation ${ }^{10,15-17}$. It has been discovered that there is a linear correlation between values of the Darunavir inhibition parameter and open-like to 
closed state population ratio ${ }^{10}$. The opening of the flap-tips is presumably essential to allow the entry of substrate to the active-site, but this conformation was not detected by crystallographic experiments due to its short life-time ${ }^{18,19}$. However, nuclear magnetic resonance (NMR) experiments have shown the flexibility of the flap-tips, which undergo sub-nanosecond time scale fluctuations ${ }^{20,21}$. According to the NMR data, all of the three possible conformations are in dynamic equilibrium, whereas the semi-open state is dominant in the ligand-free HIV-1 PR ${ }^{22-24}$. This hypothesis was further approved by Molecular dynamic (MD) simulation studies 9,13,25-27.

Darunavir is one of the HIV-1 PR inhibitors that belong to the second generation of AIDS drugs, approved by the food and drug administration (FDA), with very limited side effects ${ }^{28}$. It has been shown that the oxygen atoms of the bis-tetrahydrofuran (bis-THF) group of Darunavir (Fig. S1) can interact with the backbone and side chain atoms of Asp30 residues via hydrogen bonds ${ }^{29,30}$. As a non-peptide compound, it is proved to have a potent activity against the drug-resistant HIV-1 strains ${ }^{31,32}$. So, it has been widely used in AIDS treatment ${ }^{33-35}$. However, there are some mutations that lead to resistance of the HIV-1 PR to Darunavir. The main residues that were substituted include Val11, Val32, Leu33, Ile47, Ile50, Ile54, Gly73, Leu76, lle84 and Leu89, among which the most prevalent ones were lle47Val, Ile54Met, Ile84Val and Gly73Thr substitutions ${ }^{36,37}$.

Use of drugs against the HIV-1 PR leads to increased diversity of its mutant strains ${ }^{38}$. Recently $\sim 50$ mutations have been discovered at 30 different sites in the HIV-1 PR gene ${ }^{39}$. The mutations fall into two types: (1) mutations that occur in the active-site, which directly reduce the drug-protease interactions; (2) mutations that occur distant from the active-site, which indirectly reduce the HIV-1 PR affinity to drug by affecting the conformational dynamics of the enzyme ${ }^{36,40}$. However, some mutations may exert both effects.

This study was devoted to compare the HIV-1 PR structure, dynamics and interactions with Darunavir in mutant and wild-type strains. Our data revealed that mutations could increase the flexibility of the flaptips, make them separated and rotated with respect to each other and change active-site space, which probably help to conversion of protease's conformation from closed state to semi-open and curled states that consequently facilitate disjunction of Darunavir from the active-site, leading to the protease resistance against the Darunavir. The mechanistic knowledge from this study may provide clues for designing new inhibitors against the HIV-1 protease.

\section{Materials And Methods}

\section{System preparation}

The 3-dimentional structures of wild type complex (WT-Pr-D) (PDB:1T3R) ${ }^{41}$ and mutant HIV-1 PR complex (MUT-Pr-D) (PDB:3TTP) ${ }^{42}$ with lle47Val/lle54Met as main residual substitutions were obtained from the Protein Data Bank (PDB). Missing atoms were added to the structures using Swiss Pdb Viewer 
43. Due to the importance of the protonation state of Asp-25 (chain-B), one proton was added to its oxygen atom (OD2) in both WT-Pr-D and MUT-Pr-D ${ }^{44,45}$. Darunavir charges were obtained by using Restrained Electrostatic Potential (RESP) method ${ }^{46}$. General Amber force field (GAFF) ${ }^{47}$ parameters and the RESP charges were determined for Darunavir using the Antechamber module in the AMBER20 package ${ }^{48}$. All missing hydrogen atoms were added using the LEaP module. The WT-Pr-D and MUT-Pr-D systems were solvated with the TIP3P ${ }^{49}$ water model in the periodic boxes of size 7.71, 7.71, 5.45 ( $x, y, z$ : $\mathrm{nm})$ and 8.14, 8.14, $5.75(\mathrm{~nm})$, respectively. Distance between water molecules and box surface was determined as $10 \AA$. Then, chloride ions were added in order to neutralize the positive charges of systems. For WT complex system, 9697 TIP3P water molecules and $7 \mathrm{Cl}$ ions, and for the mutant complex system, 11533 water molecules and $3 \mathrm{Cl}$ ions were added to the simulation boxes. Then, topology files for the WTPr and MUT-Pr systems were built by GROMACS version 2019 and the ff99SB force field ${ }^{50}$.

\section{Molecular dynamics simulation}

At first step, the WT-Pr-D and MUT-Pr-D in water box were minimized by using of steepest descent minimization algorithm for 150000 steps. Constant temperature and pressure conditions were applied for both simulations ${ }^{51,52}$. The systems were equilibrated at the NVT ensemble for 150000 steps by using leap-frog integrator by time step as $2 \mathrm{fs}$ and temperature coupling was set by V-rescale algorithm. At last, temperature of systems was equilibrated at $310 \mathrm{~K}$ and followed by NPT ensemble equilibrating for another 150000 steps. The Pressure coupling and its type were determined to be Berendsen and isotropic. The pressure of systems was equilibrated at 1 bar.

For all energy minimization, NVT ensemble, NPT ensemble and MD production steps, neighbor list search was performed by using grid with $1.2 \mathrm{~nm}$ as cut-off. Lennard-Jones interactions cut-off were determined $1.2 \mathrm{~nm}$. Long-range electrostatic interactions were calculated by particle mesh ewald (PME) method ${ }^{53}$. Short range electrostatic interactions cut-off was determined as $1.2 \mathrm{~nm}$. At last, WT-Pr-D and MUT-Pr-D simulations were performed for $200 \mathrm{~ns}$. The time step for MD production was determined as $2 \mathrm{fs}$.

\section{PCA method}

Principal component analysis (PCA) method ${ }^{54}$ is often used to extract main dynamical properties from MD trajectories ${ }^{55}$. In order to perform PCA analysis, gmx-covar function of GROMACS was used to extract covariance matrix, eigenvectors and eigenvalues from 20000 frames of two system trajectories during 200 ns simulation.

The eigenvectors represent direction of motions and the eigenvalues represent mean square fluctuation on these directions ${ }^{54,56}$. The Principal components (PCs) were obtained by diagonalization of covariance matrix and calculation of the eigenvalues and eigenvectors ${ }^{54}$. At last, the gmx-anaeig program was used for projection of trajectory onto the eigenvectors to give the PCs. The first 5 PCs commonly describe $>90$ percent of system's global motions, in which the first PC contain the largest movements in the ensemble ${ }^{57}$. Our PCA analysis were based on first PC in every system. 


\section{MM-PBSA approach}

Molecular mechanics/Poisson Boltzman surface area (MM/PBSA) approach was used to calculate binding free energy between HIV-1 PR and Darunavir in both systems. The python script MMPBSA.py 58 was used to calculate the average binding energies from 2000 frames with $0.1 \mathrm{~ns}$ as interval time from both trajectories.

\section{Results And Discussion}

\section{Simulation stability}

The root mean square deviation (RMSD) for backbone atoms of proteases were analyzed in wild-type and mutant complexes to find out if the structures reached their global minimum and stable state in the 200 ns simulation time. The RMSD of 200 trajectory structures with $1 \mathrm{~ns}$ interval were analyzed (Fig. S2). The Mean RMSD and SD for backbone atoms were calculated as $0.12 \pm 0.01$ and $0.11 \pm 0.01$ for the WT-Pr-D and MUT-Pr-D, respectively. As seen, both of the mutant and wild type proteases immediately reached their stable states at RMSD value of almost $0.1 \mathrm{~nm}$ and RMSD fluctuations were small during simulation. The Darunavir/MUT-Pr-D complex showed a big structural deviation in $110 \mathrm{~ns}$ and then reached a steady state at a mean RMSD of $0.127 \pm 0.01 \mathrm{~nm}$ with small fluctuations. Darunavir/WT-Pr-D complex also reached a stable state at RMSD of $0.126 \pm 0.009 \mathrm{~nm}$. It also showed big structural deviation in earlier steps of simulation (see Fig. S2). These data indicated that both complexes reached their stable states during simulations.

\section{Comparison of the flexibility of the flap-tips}

In order to evaluate the residual flexibility, the root mean square fluctuations (RMSF) of $\mathrm{C}_{a}$ atoms were calculated for chain-A (Fig. 1a) and chain-B (Fig. 1b) of WT-Pr-D and MUT-Pr-D. The average RMSF and standard deviation (SD) values for flap-tip and active-site binding region of the MUT-Pr-D and WT-Pr-D were $0.97 \pm 0.41 \AA$ and $0.83 \pm 0.21 \AA$, respectively. As seen, mutations increased the flexibility of residues that are around Darunavir, but the residues Asp25-GIsdy26-Thr27(chain-A/B) from catalytic site remained rigid, which is in accordance with the previous experimental and theoretical studies ${ }^{59-62}$.

Figure 1c and 1d show the RMSF difference between the MUT-Pr-D and WT-Pr-D for chain A and B, respectively. Residues with an RMSF difference more than $0.50 \AA$ were considered to be highly fluctuating, which represent significant mutation-induced conformational changes. As seen, in MUT-Pr-D, there are significant decrease in the flexibility of residues Lys14-Arg20 (fulcrum of chain-A), lle36-Leu38 (flap-elbow of chain-A) and Val63-lle64 (cantilever of chain-A) (Fig. 1c). These changes can help forming of semi-open conformation in mutant strain as mentioned in Sect. 3-3. In contrast, the flexibility of the flap-tip region in chain-B (residues Gly51-Phe53) of MUT-Pr-D was remarkably increased compared with that of WT-Pr-D. It has been shown that 
increasing the flap-tip flexibility facilitate opening of the active-site gate, which consequently facilitate releasing the inhibitor from active-site ${ }^{10,12,63}$. The highly affected residues as a result of mutations in both WT-Pr and MUT-Pr complexes were illustrated by superposed 20 trajectory structures, with $10 \mathrm{~ns}$ intervals (Fig. 1e and 1f). According to these data, it can be suggested that the flap-elbow, fulcrum and cantilever regions in chain-A and the flap-tip region in chain-B experience the most conformational changes as a result of mutations.

\section{PCA analysis}

In order to extract essential dynamic motions, PCA analyses were performed ${ }^{54}$. The first principal components (pc1) was calculated by projection of both trajectories on their first eigenvector (ev1). The PCA of trajectories during 200 ns from 20000 frames is illustrated in Fig. 2, in which the cones length represents amplitude of movements and their direction represent the direction of movements.

As seen in Fig. 2, the flap-tips, cantilever (chain-A) and fulcrum (chain-A) regions of WT-Pr-D and the flaptips, flap-elbow (chain-B) and interface regions of MUT-Pr-D have larger essential dynamics than other parts that is consistent with the RMSF data (Fig. 1a and 1b). Both RMSF and PCA data showed that cantilever (chain-A) and fulcrum (chain-A) in WT-Pr-D have a higher dynamic with respect to MUT-Pr-D. As seen in Fig. 2, the movements of these regions in WT-Pr-D are directed toward the protease active-site. However, in MUT-Pr-D these movements are negligible and directed outward of the active-site (Fig. 2). Also, in WT-Pr-D, flap-elbow (chain-A) that was more flexible than that of MUT-Pr-D showed upward movement directed to closed conformation. However, in MUT-Pr-D it showed negligible movement (Fig. 2). These data showed that in wild type complex, regions surrounding active-site made its closed conformation more stable. Also, flap-tips residues in WT-Pr-D move closer to each other and to active-site (Fig. 2), but in MUT-Pr-D the flap-tip and flap-elbow of chain-B got far away from flap-tip of chain-A, downwardly on longitudinal axis (Fig. 2). The correlation between this flap-elbow and flap-tip downward movement lead to increasing of flap-tips opening and facilitate change to semi-open and open-like conformation ${ }^{10,64-67}$. Indeed, flap-tips in MUT-Pr-D show the rotation that change their conformation from two parallel planes to curled planes described as curled conformation, facilitating formation of open-like conformation (Fig. 2) ${ }^{6,25,68}$. These results indicate that the gate of active-site in MUT-Pr-D becomes susceptible to formation of open-like states compared to WT-Pr-D. In addition, the interface in MUT-Pr-D move upwardly to flap-tips and looks like it can push Darunavir outside of active-site and may help flap-tips to open (Fig. 2).

\section{Structural analysis}

In order to investigate the structural differences between WT-Pr-D and MUT-Pr-D complexes, the radial distribution function (RDF) and flap-tip distance (between $C_{a}$ of residues lle50 (chain-A)-lle50(chain-B) (Fig. 3) were examined in both complexes. The RDF and vertical distance between $C_{a}$ of residues Asp25(chain-A)- Ile50 (chain-A) and between $C_{a}$ of residues Asp25(chain-B)-lle50(chain-B) (Fig. 3) were also investigated as a greet metric to estimate the extent of flap opening ${ }^{12,69}$. 


\section{Flap tips to active-site distance measurements}

The intra-chain distances between lle50 (flap-tip) and Asp25 (active-site) were measured during the 200 ns MD simulations (see Fig. 3). The mean \pm SD distances between Asp25 and lle50 in chain-A were 14.22 \pm 0.55 and $13.94 \pm 0.43 \AA$ for MUT-Pr-D and WT-Pr-D, respectively. The most probable distances (RDF) were 14.0-14.5 $\AA$ and 13.5-14.0 $\AA$ for MUT-Pr-D and WT-Pr-D, respectively (Fig. 4a). So, in chain-A of the MUT-Pr-D, the average distance between the flap-tip and active-site was increased by $\sim 0.28 \AA$ as a result of mutation. This increase was even greater in

chain-B. The mean \pm SD distance values between Asp25 and lle50 in chain-B were $16.07 \pm 0.95$ and 15.31 $\pm 0.72 \AA$ for MUT-Pr-D and WT-Pr-D, respectively. The most probable distances were $15.8-16.5 \AA$ and 14.5-15.5 ̊ for MUT-Pr-D and WT-Pr-D, respectively (Fig. 4b). Thus, the average flap-tip to active-site distance in chain-B of the MUT-Pr-D was increased averagely by $\sim 0.76 \AA$. As seen, the upward movements of the flap-tips were increased as a result of mutations that can help to conversion of closed conformation to semi-open conformation and releasing inhibitor from its active-site binding region ${ }^{12,70}$.

\section{Flap-tip to flap-tip distance measurements}

The Average \pm SD distance between flap-tips of chain-A and chain-B were $6.20 \pm 0.71$ and $7.62 \pm 0.73 \AA$ in MUT-Pr-D and WT-Pr-D, respectively. The most probable distances were 5.8-6.5 $\mathrm{A}$ and 7.5-8 $\AA$ for MUT-PrD and WT-Pr-D, respectively (Fig. 4c). Based on previous reports, the flap-tips separation more than $10 \AA$ was considered as open conformation, from 6 to $10 \AA$ as semi-open conformation and the separation of less than $6 \AA$ as the closed structure ${ }^{8,10,12}$. As seen, the flap-tip to flap-tip average distance was about $1.42 \AA$ smaller in MUT-Pr-D. Because lle50 in chains-A and B were located in the cap of flap-tips, when the flap-tips got far away from each other along the longitudinal axis, these residues become closer to each other (Fig. 3). However, in our nano second duration simulation, flap-tips conversion from closed conformation to semi-open have not been shown directly because it acquires micro second to millisecond time to occur as NMR experiments have revealed [22-24].

\section{Hydrophobic interactions stabilize the closed conformation of flap-tips}

In ligand-bonded WT-Pr, hydrophobic interactions between Ile50(chain-A) and its adjacent residues Ile47(chain-B)/Ile54(chain-B) as well as hydrophobic interactions between lle50(chain-B) and its adjacent residues Ile47(chain-A)/lle54(chain-A) (Fig. 5) play an important role in retention of the active-site in closed conformation, which consequently traps Darunavir in the active-site ${ }^{71-73}$. Previous experimental analysis on the extensive statistical community that suffer from AIDS disease have revealed that lle47val and lle54Met mutations are most prevalent in resistant strains to Darunavir ${ }^{74}$. So, this type of Mut-Pr was selected and the hydrophobic interactions during 200 ns MD simulations were compared in MUT-PrD and WT-Pr-D. For this purpose, the Radial Distribution Function and distance between Ca atoms of Ile50(chain-B) and Ile47(chain-A)/ Ile54(chain-A) (Fig. S3 and Fig. 5) and distance between lle50(chain-A) 
with Ile47(chain-B)/ Ile54(chain-B) in WT-Pr and MUT-Pr-D, in which lle47 was substituted by Val and lle54 was substituted by Met, were calculated (Fig. S4 and Fig. 5)

The average distance \pm SD between Ile50(B) and Val47(A) in MUT-Pr-D and between lle50(chain-B) and lle47(chain-A) in WT-Pr-D were calculated $9.25 \pm 0.88 \AA$ and $8.82 \pm 0.51 \AA$, respectively. The most probable distances were calculated as 8.1-10 $\AA$ and 8.3-9.2 $\AA$ for MUT-Pr-D and WT-Pr-D, respectively (Fig. S3-a). As seen, in MUT-Pr-D, the average distance was increased $\sim 0.43 \AA$ compared to that of WT-Pr-D. The Average Distance \pm SD between lle50 (chain-B) and Met54(chain-A) in MUT-Pr-D and between lle50(chainB) and Ile54(chain-A) in WT-Pr-D were calculated to be $7.02 \pm 0.55$ and $7.48 \pm 0.63 \AA$, respectively. The most probable distances were calculated as $6.5-7.2 \AA$ and 7.3-7.8 $\AA$ for MUT-Pr-D and WT-Pr-D, respectively. So, in MUT-Pr-D, the average distance decreases about $0.46 \AA$ relative to WT-Pr-D (Fig. S3-b).

The Average distance \pm SD between lle50 (chain-A) and Val47(chain-B) in MUT-Pr-D and between Ile50(chain-A) and Ile47(chain-B) in WT-Pr-D were calculated to be $9.29 \pm 0.91$ and $8.10 \pm 0.53 \AA$, respectively. The most probable distances were calculated as $8.3-8.7,9.9-10.3 \AA$ and $7.7-8.2 \AA$ for MUTPr-D and WT-Pr-D, respectively (Fig. S4-a), showing that average distance increased about $1.19 \AA$ with respect to WT-Pr-D due to mutations. The Average distance \pm SD between lle50(chain-A) and Met54(chainB) in MUT-Pr-D and between Ile50(chain-A) and Ile54(chain-B) in WT-Pr-D were calculated to be $8.40 \pm$ $0.75 \AA$ and $7.41 \pm 0.35 \AA$, respectively. The most probable distances were calculated as 8-8.5 $\AA$ and $7.2-$

$7.6 \AA$ for MUT-Pr-D and WT-Pr-D, respectively. So, this distance increased about $1 \AA$ respect to WT-Pr-D due to mutations (Fig. S4-b). however, distance between Ile50(chain-B) and Met54(chain-A) in MUT-Pr-D was decreased but this can't increase hydrophobic interaction between flap-tips because MET isn't a hydrophobic residue (Fig. 5 and Fig. S3-b). Indeed, except lle50 (chain-B) and Met54(chain-A), distances between other mentioned three-pair hydrophobic residues increased in MUT-Pr-D compared to that in WTPr-D. So, the flap-tips separation relative to each other occurs due to the reduction of hydrophobic interactions that consequently forms semi-open conformation (Fig. 5) ${ }^{72}$. As a result, binding site gateway could open more easy in MUT-Pr-D than WT-Pr-D [10, 12].

\section{Comparing the interactions in WT-Pr-D and MUT-Pr-D systems}

In order to compare the binding strength between Darunavir and WT-Pr or MUT-Pr, the binding energies were calculated by MM-PBSA approach ${ }^{75}$ (see data in Table 1). According to the data presented in Table 1, the part of binding energy related to electrostatic interactions $\left(\Delta \mathrm{E}_{\text {electrostatic }}\right)$ were almost the same in both complexes. the Polar solvation energy $\left(\Delta G_{\text {Polar-solvation }}\right)$ and non-polar solvation energy $\left(\Delta \mathrm{G}_{\text {SASA }}\right)$ showed little changes, so that in the WT-Pr- D, polar interactions were a little more unstable and non-polar interactions were a little more stable than that in MUT-Pr-D. Thus, these interactions had same effect on total binding energy in both complexes too. However, van der Waals interactions between the protease and Darunavir have been decreased in MUT-Pr-D. So, the protease-Darunavir binding energy in MUT-Pr-D was smaller than that of WT-Pr-D by about $20 \mathrm{~kJ} / \mathrm{mol}$ (Table 1). This indicates that the 
mutations have reduced the binding strength between the protease and Darunavir in the MUT-Pr-D system by decreasing van der waals interactions.

Table 1

The binding energy components between the protease and Darunavir in WT-Pr-D and MUT-Pr-D systems.

\begin{tabular}{|llllll|}
\hline System & $\Delta \mathrm{E}_{\mathrm{vdw}}$ & $\begin{array}{l}\Delta \mathrm{E}_{\text {electrostatic }} \\
(\mathrm{Kcal} / \mathrm{mol})\end{array}$ & $\begin{array}{l}\Delta \mathrm{G}_{\text {Polar-solvation }} \\
(\mathrm{Kcal} / \mathrm{mol})\end{array}$ & $\begin{array}{l}\Delta \mathrm{G}_{\mathrm{SASA}} \\
(\mathrm{Kcal} / \mathrm{mol})\end{array}$ & $\begin{array}{l}\Delta \mathrm{G}_{\mathrm{MMPBSA}} \\
(\mathrm{Kcal} / \mathrm{mol})\end{array}$ \\
\hline WI-Pr-D & $-280.71+/-26.0$ & $-88.52+/-14.9$ & $252.40+/-17.3$ & $-26.258+/-$ & $-143.08+/-$ \\
\hline $\begin{array}{l}\text { MUT- } \\
\text { Pr-D }\end{array}$ & $-260.91+/-16.3$ & $-88.82+/-27.7$ & $250.59+-30.1$ & $-24.44+/-$ & $123.583+/-18.2$ \\
\hline
\end{tabular}

All of the energies are in $\mathrm{Kcal} / \mathrm{mol}$. Total binding energies $\left(\Delta \mathrm{G}_{\text {MMPBSA}}\right.$ : Molecular mechanics/PoissonBoltzmann Surface Area) obtained from sum of the $\Delta \mathrm{E}_{\mathrm{vdw}}$ (van der Waals interaction energy),

$\Delta \mathrm{E}_{\text {electrostatic }}$ (Electrostatic interaction energy), $\Delta \mathrm{G}_{\text {Polar-solvation }}$ (Polar solvation energy) and $\Delta \mathrm{G}_{\mathrm{SASA}}$ (Non-Polar solvation energy) energies.

\section{Hydrogen bonds between Darunavir and HIV-1 proteases}

Hydrogen bonds pattern between Darunavir and HIV-1 PR are important and can affect inhibition ${ }^{16,76,77}$. In order to investigate the effect of mutation on these interactions, hydrogen bonds between Darunavir and HIV-1 PR from all 20000 frames in both trajectories were analyzed by Hbonanza ${ }^{78}$ program. Figure 6 shows the hydrogen bonds data, shown by color gradient, in which white color dashed lines represent the hydrogen bonds appearing at least in 10 percent of whole simulation time and green color dashed lines represent those appearing in 100 percent of entire 200 ns simulation time. Only hydrogen bonds in which the distance between hydrogen donor and acceptor atoms were $3.5 \AA$ and angle of Donor-H-acceptor atoms were between $0-30^{\circ}$ were considered. Figure 6 shows the comparison of hydrogen bond pattern in WT-Pr-D with MUT-Pr-D. As seen, the Asp25(chain-A/B) are very important residues in the catalytic site, locating near each other, forming strong hydrogen bond interactions with each other in whole simulation time in both WT-Pr-D and MUT-Pr-D. Both sidechain oxygen atoms of Asp25(chain-A/OD1, OD2) are involved in interaction with two Darunavir's atoms $(018, N 20)$ in WT-Pr-D by hydrogen bond interactions, while in MUT-Pr-D only one oxygen atom (OD2) of Asp25(chain-A) is involved in hydrogen bond interaction with only one Darunavir atom (018). This is because of Darunavir rotation in the active-site of MUT-Pr-D (by $180^{\circ}$ ) with respect to WT-Pr-D. Also, in WT-Pr-D, hydrogen bond between N21 atom of Darunavir and Asp30 (chain-B) in backbone of protease was formed in whole trajectory. However, in MUTPr-D, hydrogen bond between N21 atom of Darunavir and backbone of Asp30(chain-A) was formed almost half of trajectory's frames. These results indicate that in WT-Pr-D, strongest hydrogen bond interactions are involved in binding of Darunavir to the active-site and catalytic residues.

\section{Radius of gyration}


In order to assess the compactness of the protease active-site cavity, the radius of gyration $(\mathrm{g}(\mathrm{r}))$ of backbone atoms was calculated for residues 23-32, 46-54 and 78-87 in both chains (Fig. S5). As seen, the average $\pm S D g(r)$ values during first 110 ns simulation time for MUT-Pr-D and WT-Pr-D were $10.50 \pm$ $0.09 \AA$ and $10.62 \pm 0.10 \AA$ respectively. The average $g(r)$ in WT-Pr-D was $\sim 0.12 \AA$ larger than that of MUTPr-D. So, during the first $110 \mathrm{~ns}$, the active-site of MUT-Pr-D was more compact than of WT-Pr-D. However, after $110 \mathrm{~ns}$, the average $\mathrm{g}(\mathrm{r})$ and standard deviation for MUT-Pr-D and WT-Pr-D were calculated as 10.54 $\pm 0.07 \AA$ and $10.52 \pm 0.06 \AA$, respectively. Thus, the compactness of the MUT-Pr-D active-site decreased a little, while in WT-Pr-D it became more compact.

So, at last $90 \mathrm{~ns}$, respect to the beginning of simulation, the active-site cavity in the WT-Pr-D was decreased about $0.12 \AA$ but it was increased about $0.04 \AA$ in MUT-Pr-D. As a result, compactness of WTPr- D become increased but compactness of MUT-Pr-D become a little decreased these results are in agreement with other structural and dynamical analysis (Fig. S2-a).

\section{Conclusion}

The principal components and fluctuation analyses showed that in MUT-Pr-D, the flap-tips get away from each other, curl upwardly and become more flexible in chain-B. Also, movement correlation between Flaptip(chain-B) and Flap-Elbow(chain-B) in MUT-Pr-D was seen. All these evidences confirm that as a result of mutation, the formation of semi-open conformation become more probable and the closed conformation become unstable. Based on the distance and RDF analysis, it was also shown that the average distance of flap-tips from each other and from the active-site increased in MUT-Pr-D, leading to conversion from closed to a semi-open conformation more probably. Increase of flap-tip(chain-A) to flap-

tip (chain-B) distance occurred as a result of decreasing hydrophobic interactions in MUT-Pr-D. PCA and $g(r)$ analysis showed that the volume of active-site in MUT-Pr-D becomes a little larger. This is because in WT-Pr-D, the fulcrum, cantilever and flap-tips move toward the active-site core, while in MUT-Pr-D, these movements were slightly outward from the core of active-site. Thus, the MUT-Pr-D conformation become near to semi-open conformation. At last, hydrogen bonds analysis indicated that the hydrogen bonds between Darunavir and the active-site and catalytic residues of protease were weaker in MUT-Pr-D. Binding energy calculations also approved that binding strength between Darunavir and MUT-Pr decrease as a result of mutations. Briefly, it can be concluded that resistance of MUT-Pr to Darunavir occurred due to changing HIV-1 PR structure and dynamic as a result of mutation.

\section{Declarations}

\section{Acknowledgements}

The authors would like to acknowledge all of their colleagues in the Department of Biophysics, Faculty of Biological Sciences, Tarbiat Modares University, Tehran, Iran for their help.

\section{Author contributions}


Y. S. carried out data gathering, analysis, validation, visualization and wrote the manuscript. E. B. participated in data analysis, visualization and writing the original draft. P. A. contributed in data analysis and validation. A. H. K. contributed in project administration and validation. S.S. contributed in project supervision and completing the draft. All authors reviewed the final draft of manuscript.

\section{Competing interests}

The authors declare no competing interests.

\section{References}

1. Sillapachaiyaporn, C. \& Chuchawankul, S. HIV-1 protease and reverse transcriptase inhibition by tiger milk mushroom (Lignosus rhinocerus) sclerotium extracts: In vitro and in silico studies. Journal of traditional and complementary medicine. 10, 396-404 (2020).

2. Ghosh, A. K., Osswald, H. L. \& Prato, G. Recent Progress in the Development of HIV-1 Protease Inhibitors for the Treatment of HIV/AIDS. Journal of medicinal chemistry. 59, 5172-5208 (2016).

3. Wang, R. G., Zhang, H. X. \& Zheng, Q. C. Revealing the binding and drug resistance mechanism of amprenavir, indinavir, ritonavir, and nelfinavir complexed with HIV-1 protease due to double mutations G48T/L89M by molecular dynamics simulations and free energy analyses. Physical Chemistry Chemical Physics. 22, 4464-4480 (2020).

4. Mager, P. P. The active site of HIV-1 protease. Medicinal research reviews. 21, 348-353 (2001).

5. Sahin, K. Investigation of novel indole-based HIV-1 protease inhibitors using virtual screening and text mining. Journal of Biomolecular Structure and Dynamics,1-11(2020).

6. Yu, Y. et al. Structural insights into HIV-1 protease flap opening processes and key intermediates. RSC advances. 7, 45121-45128 (2017).

7. Tie, Y. et al. High resolution crystal structures of HIV-1 protease with a potent non-peptide inhibitor (UIC-94017) active against multi-drug-resistant clinical strains. Journal of molecular biology. 338, 341-352 (2004).

8. Sk, M. F., Roy, R. \& Kar, P. Exploring the potency of currently used drugs against HIV-1 protease of subtype D variant by using multiscale simulations. Journal of Biomolecular Structure and Dynamics,1-16(2020).

9. Apoorva, B. \& Sasidhar, Y. U. Inhibition of the activity of HIV-1 protease through antibody binding and mutations probed by molecular dynamics simulations. Scientific Reports (Nature Publisher Group)10 (2020).

10. Liu, Z. et al. Darunavir-Resistant HIV-1 Protease Constructs Uphold a Conformational Selection Hypothesis for Drug Resistance. Viruses. 12, 1275 (2020).

11. Huang, Y. M., Raymundo, M. A. V., Chen, W. \& Chang, C. -e. A. Mechanism of the association pathways for a pair of fast and slow binding ligands of HIV-1 protease. Biochemistry. 56, 1311-1323 (2017). 
12. Badaya, A. \& Sasidhar, Y. U. Inhibition of the activity of HIV-1 protease through antibody binding and mutations probed by molecular dynamics simulations. Scientific reports. 10, 1-15 (2020).

13. Hornak, V., Okur, A., Rizzo, R. C. \& Simmerling, C. HIV-1 protease flaps spontaneously open and reclose in molecular dynamics simulations. Proceedings of the National Academy of Sciences 103, 915-920(2006).

14. Hornak, V. \& Simmerling, C. Targeting structural flexibility in HIV-1 protease inhibitor binding. Drug discovery today. 12, 132-138 (2007).

15. Carter, J. D. et al. Characterizing solution surface loop conformational flexibility of the GM2 activator protein. The Journal of Physical Chemistry B. 118, 10607-10617 (2014).

16. Huang, X. et al. The role of select subtype polymorphisms on HIV-1 protease conformational sampling and dynamics. Journal of Biological Chemistry. 289, 17203-17214 (2014).

17. Liu, Z. et al. Pulsed EPR characterization of HIV-1 protease conformational sampling and inhibitorinduced population shifts. Physical Chemistry Chemical Physics. 18, 5819-5831 (2016).

18. Navia, M. A. et al. Three-dimensional structure of aspartyl protease from human immunodeficiency virus HIV-1. Nature. 337, 615-620 (1989).

19. Lapatto, R. et al. X-ray analysis of HIV-1 proteinase at $2.7 \AA$ resolution confirms structural homology among retroviral enzymes. Nature. 342, 299-302 (1989).

20. Deshmukh, L., Tugarinov, V., Louis, J. M. \& Clore, G. M. Binding kinetics and substrate selectivity in HIV-1 protease - Gag interactions probed at atomic resolution by chemical exchange NMR. Proceedings of the National Academy of Sciences 114, E9855-E9862(2017).

21. Roche, J., Louis, J. M. \& Bax, A. Conformation of inhibitor-free HIV-1 protease derived from nmr spectroscopy in a weakly oriented solution. ChemBioChem. 16, 214-218 (2015).

22. Freedberg, D. I. et al. Rapid structural fluctuations of the free HIV protease flaps in solution: relationship to crystal structures and comparison with predictions of dynamics calculations. Protein science. 11, 221-232 (2002).

23. Ishima, R., Freedberg, D. I., Wang, Y. X., Louis, J. M. \& Torchia, D. A. Flap opening and dimer-interface flexibility in the free and inhibitor-bound HIV protease, and their implications for function. Structure. 7, 1047-1012 (1999).

24. Katoh, E. et al. A solution NMR study of the binding kinetics and the internal dynamics of an HIV-1 protease-substrate complex. Protein Sci. 12, 1376-1385 (2003).

25. Scott, W. R. \& Schiffer, C. A. Curling of flap tips in HIV-1 protease as a mechanism for substrate entry and tolerance of drug resistance. Structure. 8, 1259-1265 (2000).

26. Tozzini, V. \& McCammon, J. A. A coarse grained model for the dynamics of flap opening in HIV-1 protease. Chem. Phys. Lett. 413, 123-128 (2005).

27. Gupta, S. \& Senapati, S. Mechanism of inhibition of drug-resistant HIV-1 protease clinical isolates by TMC310911: A molecular dynamics study. Journal of Molecular Structure. 1198, 126893 (2019). 
28. Darwish, I. A. et al. Darunavir: A comprehensive profile. Profiles of Drug Substances, Excipients, and Related Methodology. 46, 1-50 (2020).

29. Kovalevsky, A. Y. et al. Effectiveness of nonpeptide clinical inhibitor TMC-114 on HIV-1 protease with highly drug resistant mutations D30N, I50V, and L90M. Journal of medicinal chemistry. 49, 13791387 (2006).

30. Orkin, C. et al. Week 96 results of a phase 3 trial of Darunavir/cobicistat/emtricitabine/tenofovir alafenamide in treatment-naive HIV-1 patients. Aids. 34, 707-718 (2020).

31. Kovalevsky, A. Y., Louis, J. M., Aniana, A., Ghosh, A. K. \& Weber, I. T. Structural evidence for effectiveness of Darunavir and two related antiviral inhibitors against HIV-2 protease. Journal of molecular biology. 384, 178-192 (2008).

32. Ghosh, A. K., Dawson, Z. L. \& Mitsuya, H. Darunavir, a conceptually new HIV-1 protease inhibitor for the treatment of drug-resistant HIV. Bioorganic \& medicinal chemistry. 15, 7576-7580 (2007).

33. Santos, A. M. R. et al. Long-term virological effectiveness with Darunavir/ritonavir-based salvage therapy in people living with HIV/AIDS from São Paulo, Brazil. International journal of STD \& AIDS. 31, 967-975 (2020).

34. Navarro, J. et al. Effectiveness of boosted Darunavir plus rilpivirine in patients with long-lasting HIV-1 infection: DARIL study. Journal of Antimicrobial Chemotherapy. 75, 1955-1960 (2020).

35. Chow, W. et al. Treatment Patterns and Predictors of Adherence in HIV Patients Receiving Single-or Multiple-Tablet Darunavir, Cobicistat, Emtricitabine, and Tenofovir Alafenamide. Patient Preference and Adherence. 14, 2315-2326 (2020).

36. Johnson, V. A. et al. Update of the drug resistance mutations in HIV-1: december 2009. Clin Infect Dis. 47, 266-285 (2008).

37. Tremblay, C. L. Combating HIV resistance-focus on Darunavir. Therapeutics and clinical risk management. 4, 759 (2008).

38. Carter, J. D. et al. Effects of PRE and POST therapy drug-pressure selected mutations on HIV-1 protease conformational sampling. FEBS letters. 588, 3123-3128 (2014).

39. Wensing, A. M. et al. 2019 update of the drug resistance mutations in HIV-1. Topics in antiviral medicine. 27, 111 (2019).

40. Meher, B. R. \& Wang, Y. Interaction of I50V mutant and I50L/A71V double mutant HIV-protease with inhibitor TMC114 (Darunavir): molecular dynamics simulation and binding free energy studies. The Journal of Physical Chemistry B. 116, 1884-1900 (2012).

41. Surleraux, D. L. et al. Discovery and selection of TMC114, a next generation HIV-1 protease inhibitor. Journal of medicinal chemistry. 48, 1813-1822 (2005).

42. Kožíšek, M. et al. Thermodynamic and structural analysis of HIV protease resistance to Darunaviranalysis of heavily mutated patient-derived HIV-1 proteases. The FEBS journal. 281, 1834-1847 (2014). 
43. Guex, N. \& Peitsch, M. C. SWISS-MODEL and the Swiss-Pdb Viewer: an environment for comparative protein modeling. electrophoresis 18, 2714-2723(1997).

44. Hyland, L. J., Tomaszek, T. A. Jr \& Meek, T. D. Human immunodeficiency virus-1 protease. 2. Use of $\mathrm{pH}$ rate studies and solvent kinetic isotope effects to elucidate details of chemical mechanism. Biochemistry. 30, 8454-8463 (1991).

45. Wang, Y. X. et al. Solution NMR evidence that the HIV-1 protease catalytic aspartyl groups have different ionization states in the complex formed with the asymmetric drug KNI-272. Biochemistry. 35, 9945-9950 (1996).

46. Bayly, C. I., Cieplak, P., Cornell, W. \& Kollman, P. A. A well-behaved electrostatic potential based method using charge restraints for deriving atomic charges: the RESP model. The Journal of Physical Chemistry. 97, 10269-10280 (1993).

47. Wang, J., Wolf, R. M., Caldwell, J. W., Kollman, P. A. \& Case, D. A. Development and testing of a general amber force field. Journal of computational chemistry. 25, 1157-1174 (2004).

48. Case, D. A. et al. Amber 2020(2020).

49. Jorgensen, W. L., Chandrasekhar, J., Madura, J. D., Impey, R. W. \& Klein, M. L. Comparison of simple potential functions for simulating liquid water. The Journal of chemical physics. 79, 926-935 (1983).

50. Koes, D. R. \& Vries, J. K. Evaluating amber force fields using computed NMR chemical shifts. Proteins: Structure, Function, and Bioinformatics. 85, 1944-1956 (2017).

51. Berendsen, H. J., Postma, J., van Gunsteren, W. F., DiNola, A. \& Haak, J. R. Molecular dynamics with coupling to an external bath. The Journal of chemical physics. 81, 3684-3690 (1984).

52. Ryckaert, J. P., Ciccotti, G. \& Berendsen, H. J. Numerical integration of the cartesian equations of motion of a system with constraints: molecular dynamics of n-alkanes. Journal of computational physics. 23, 327-341 (1977).

53. Essmann, U. et al. A smooth particle mesh Ewald method. The Journal of chemical physics. 103, 8577-8593 (1995).

54. David, C. C. \& Jacobs, D. J. in Protein dynamics193-226(Springer, 2014).

55. Bahar, I., Atilgan, A. R., Demirel, M. C. \& Erman, B. Vibrational dynamics of folded proteins: significance of slow and fast motions in relation to function and stability. Physical review letters. $\mathbf{8 0}$, 2733 (1998).

56. Du, Q. S., Jiang, Z. Q., He, W. Z., Li, D. P. \& Chou, K. C. Amino acid principal component analysis (AAPCA) and its applications in protein structural class prediction. Journal of Biomolecular Structure and Dynamics. 23, 635-640 (2006).

57. Maisuradze, G. G., Liwo, A. \& Scheraga, H. A. Principal component analysis for protein folding dynamics. Journal of molecular biology. 385, 312-329 (2009).

58. Kumari, R., Kumar, R., Consortium, O. S. D. D. \& Lynn, A. g_mmpbsaî-, A GROMACS tool for highthroughput MM-PBSA calculations. Journal of chemical information and modeling. 54, 1951-1962 
(2014).

59. Freedberg, D. I. et al. Flexibility and function in HIV protease: Dynamics of the HIV-1 protease bound to the asymmetric inhibitor kynostatin 272 (KNI-272). Journal of the American Chemical Society. 120, 7916-7923 (1998).

60. Hou, T. \& Yu, R. Molecular dynamics and free energy studies on the wild-type and double mutant HIV1 protease complexed with amprenavir and two amprenavir-related inhibitors: mechanism for binding and drug resistance. Journal of medicinal chemistry. 50, 1177-1188 (2007).

61. Zoete, V., Michielin, O. \& Karplus, M. Relation between sequence and structure of HIV-1 protease inhibitor complexes: a model system for the analysis of protein flexibility. Journal of molecular biology. 315, 21-52 (2002).

62. Agniswamy, J., Louis, J. M., Roche, J., Harrison, R. W. \& Weber, I. T. Structural studies of a rationally selected multi-drug resistant HIV-1 protease reveal synergistic effect of distal mutations on flap dynamics. PloS one. 11, e0168616 (2016).

63. Nicholson, L. K. et al. Flexibility and function in HIV-1 protease. Nature structural biology. 2, 274-280 (1995).

64. Ung, P. M. U., Dunbar, J. B. Jr, Gestwicki, J. E. \& Carlson, H. A. An allosteric modulator of HIV-1 protease shows equipotent inhibition of wild-type and drug-resistant proteases. Journal of medicinal chemistry. 57, 6468-6478 (2014).

65. Kunze, J. et al. Targeting dynamic pockets of HIV-1 protease by structure-based computational screening for allosteric inhibitors. Journal of chemical information and modeling. 54, 987-991 (2014).

66. Perryman, A. L., Lin, J. H. \& McCammon, J. A. HIV-1 protease molecular dynamics of a wild-type and of the V82F/I84V mutant: Possible contributions to drug resistance and a potential new target site for drugs. Protein Sci. 13, 1108-1123 (2004).

67. Perryman, A. L., Lin, J. H. \& McCammon, J. A. Restrained molecular dynamics simulations of HIV-1 protease: The first step in validating a new target for drug design. Biopolymers: Original Research on Biomolecules. 82, 272-284 (2006).

68. de Vera, I. M. S. et al. Elucidating a relationship between conformational sampling and drug resistance in HIV-1 protease. Biochemistry. 52, 3278-3288 (2013).

69. Okimoto, N., Kitayama, K., Hata, M., Hoshino, T. \& Tsuda, M. Molecular dynamics simulations of a complex of HIV-1 protease and substrate: substrate-dependent efficiency of catalytic activity. Journal of Molecular Structure: THEOCHEM. 543, 53-63 (2001).

70. Ding, F., Layten, M. \& Simmerling, C. Solution structure of HIV-1 protease flaps probed by comparison of molecular dynamics simulation ensembles and EPR experiments. Journal of the American Chemical Society. 130, 7184-7185 (2008).

71. Lange, O. F. \& Grubmüller, H. Generalized correlation for biomolecular dynamics. Proteins: Structure, Function, and Bioinformatics. 62, 1053-1061 (2006). 
72. Yu, Y., Wang, J., Shao, Q., Shi, J. \& Zhu, W. Effects of drug-resistant mutations on the dynamic properties of HIV-1 protease and inhibition by Amprenavir and Darunavir. Scientific reports. $5,1-14$ (2015).

73. Pawar, S. et al. Structural studies of antiviral inhibitor with HIV-1 protease bearing drug resistant substitutions of V32I, 147V and V82I. Biochemical and biophysical research communications. 514, 974-978 (2019).

74. Wheeler, W. H. et al. Prevalence of transmitted drug resistance associated mutations and HIV-1 subtypes in new HIV-1 diagnoses, US-2006. Aids. 24, 1203-1212 (2010).

75. Genheden, S. \& Ryde, U. The MM/PBSA and MM/GBSA methods to estimate ligand-binding affinities. Expert opinion on drug discovery. 10, 449-461 (2015).

76. Huang, X. et al. Inhibitor-induced conformational shifts and ligand-exchange dynamics for HIV-1 protease measured by pulsed EPR and NMR spectroscopy. The Journal of Physical Chemistry B. 116, 14235-14244 (2012).

77. Sayer, J. M., Liu, F., Ishima, R., Weber, I. T. \& Louis, J. M. Effect of the active site D25N mutation on the structure, stability, and ligand binding of the mature HIV-1 protease. Journal of Biological Chemistry. 283, 13459-13470 (2008).

78. Durrant, J. D. \& McCammon, J. A. HBonanza: a computer algorithm for molecular-dynamicstrajectory hydrogen-bond analysis. Journal of Molecular Graphics and Modelling. 31, 5-9 (2011).

\section{Figures}


a

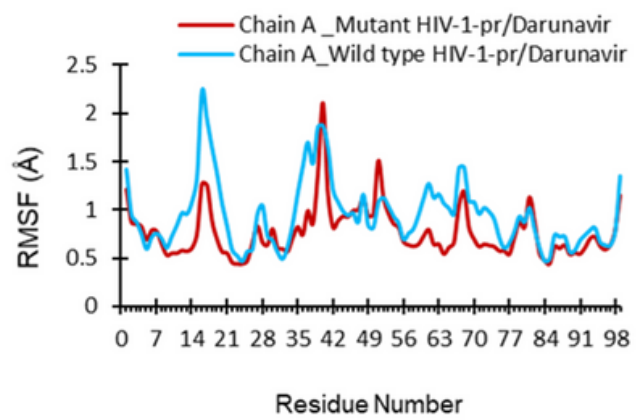

C

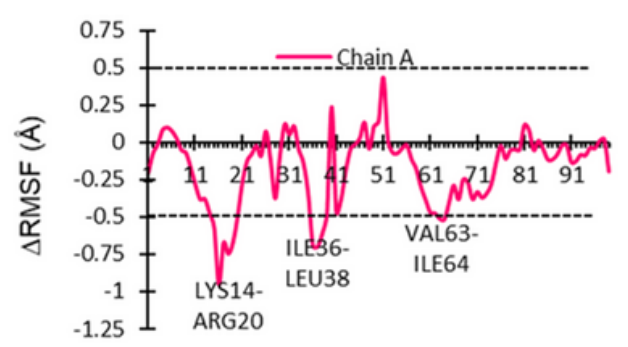

b

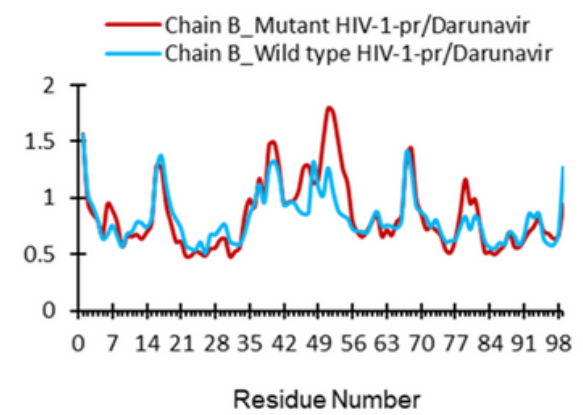

d

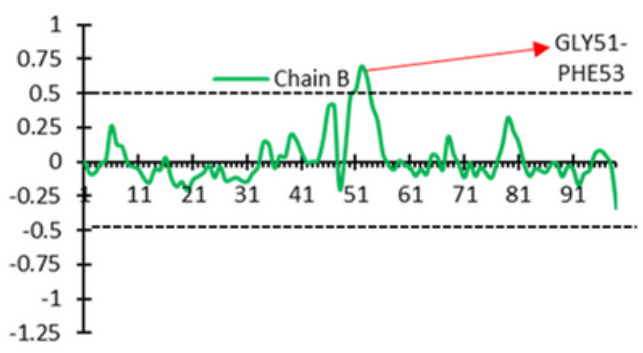

e

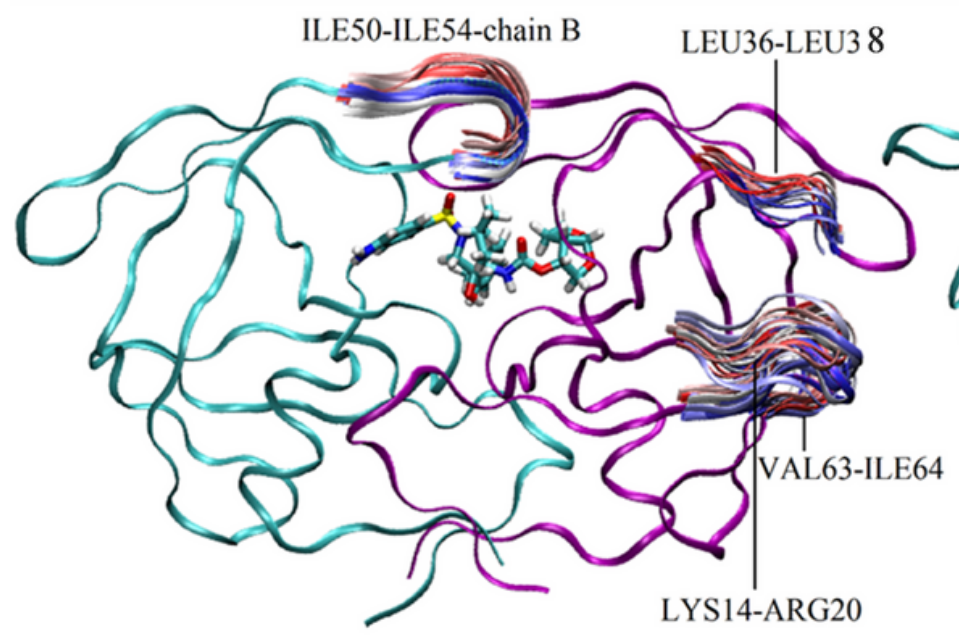

f

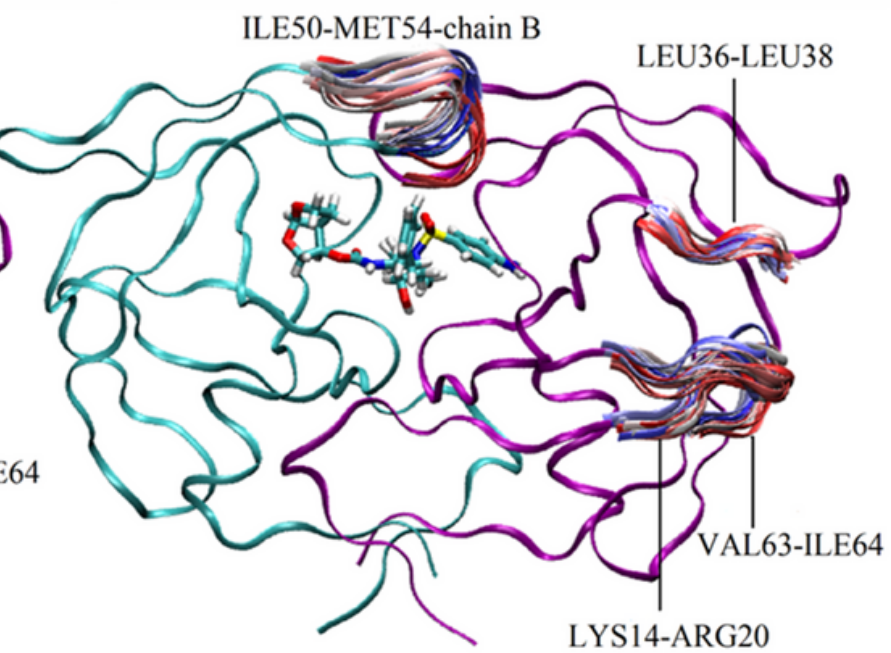

Figure 1

Ca root mean square fluctuation (RMSF) values for chain-A (a) and chain-B (b) of WT-Pr-D and MUT-Pr-D and difference of RMSF values between MUT-Pr-D and WT-Pr-D for chain-A (c) and chain-B (d). Residues with absolute difference larger than $0.5 \AA$ (exceeding the black cutoff lines) are labeled. The 20 superimposed Trajectories snapshots of WT-Pr-D (e) and MUT-Pr-D (f) with 10 ns intervals were obtained and only regions that had remarkable alternation in flexibility due to mutations were illustrated. 


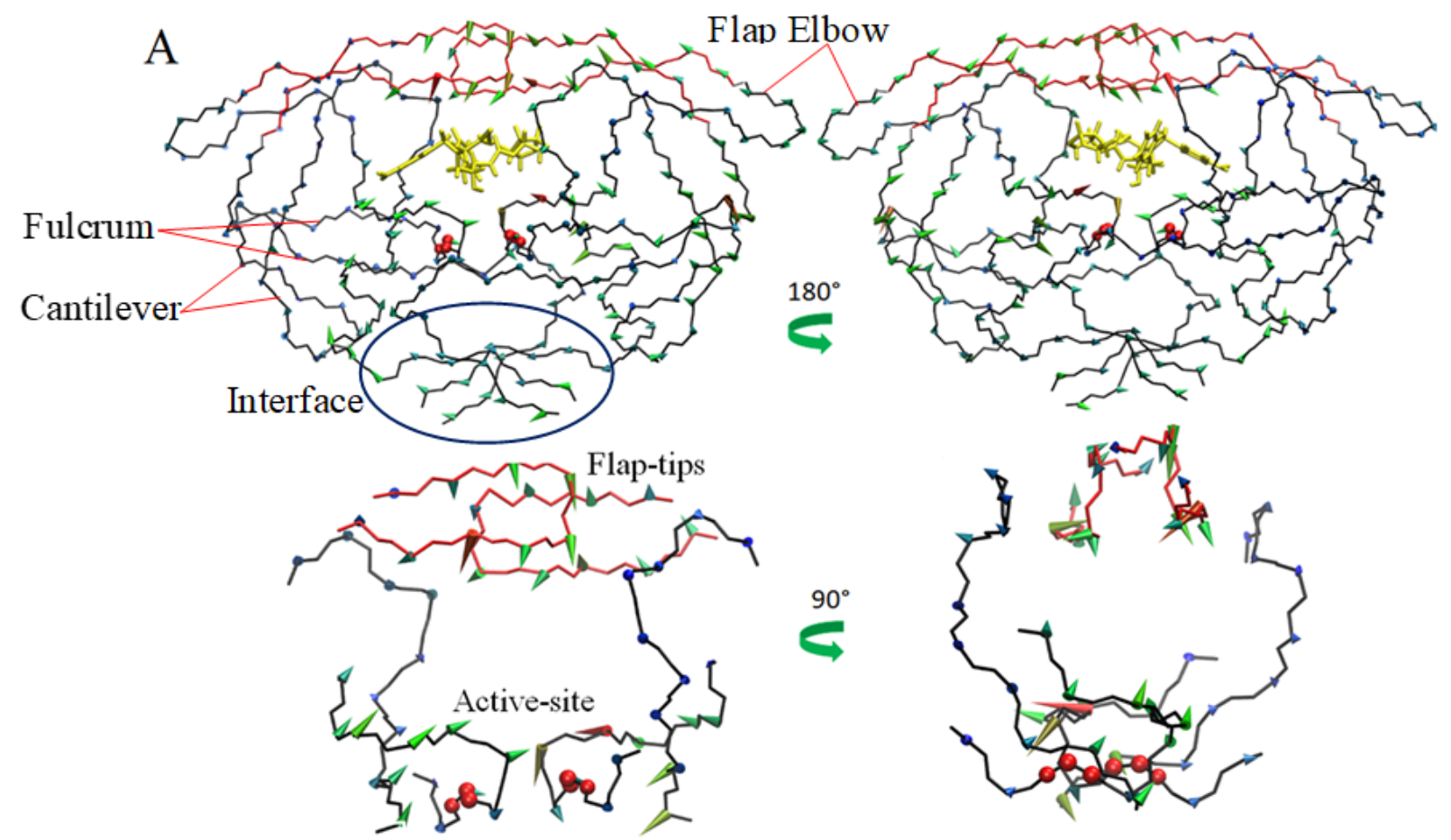

Chain-A
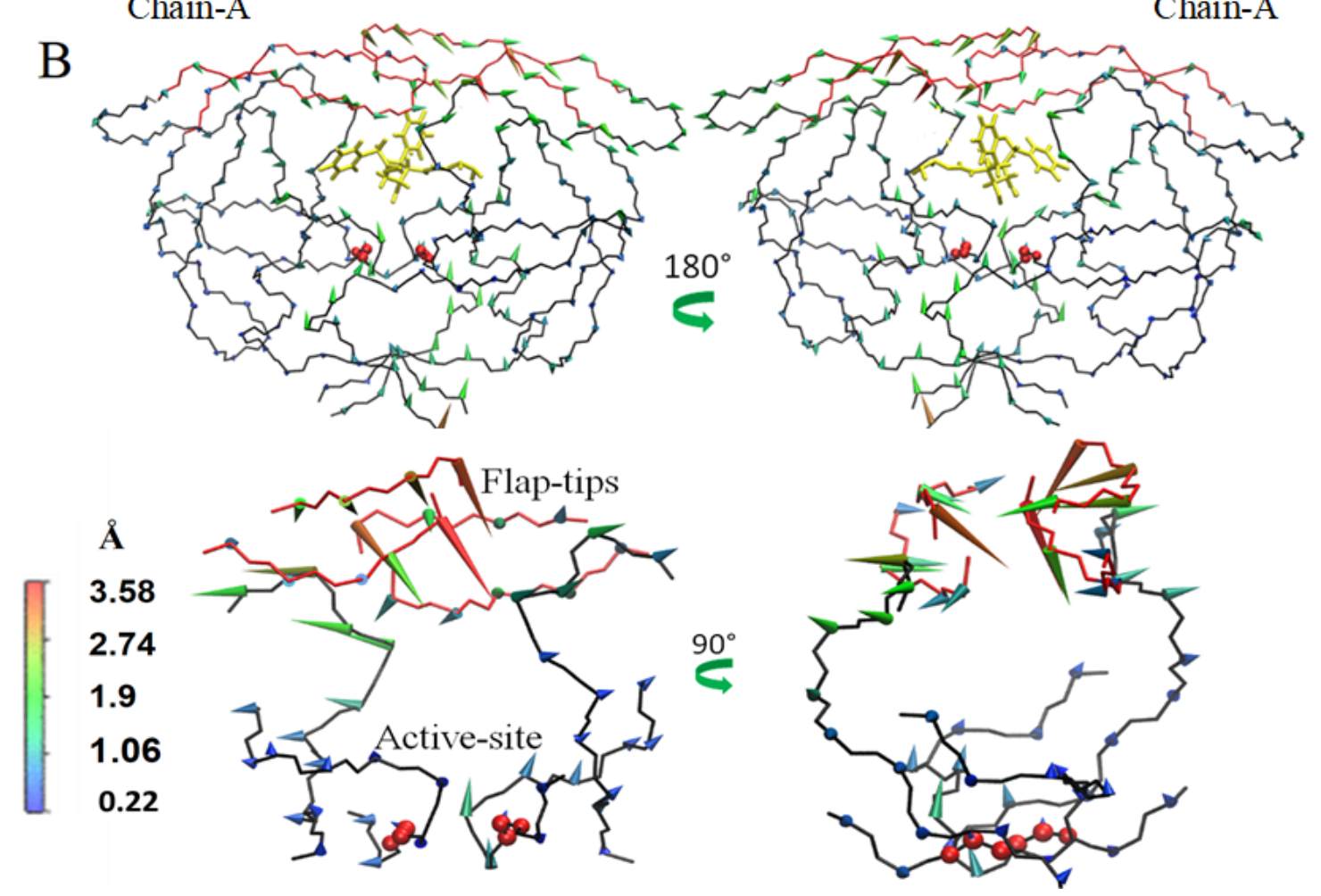

Figure 2

The Ca essential dynamic of WT-Pr-D (A) and MUT-Pr-D (B) based on their first eigenvectors are shown. Darunavir is indicated by yellow, flap-tip regions are seen in red, and backbone atoms of ASP25, the catalytic residue, in both chains are shown by red spheres. The direction of the cones describes the direction of motions and their length are correlated with magnitude of motions, that also indicated by color gradient scale. 


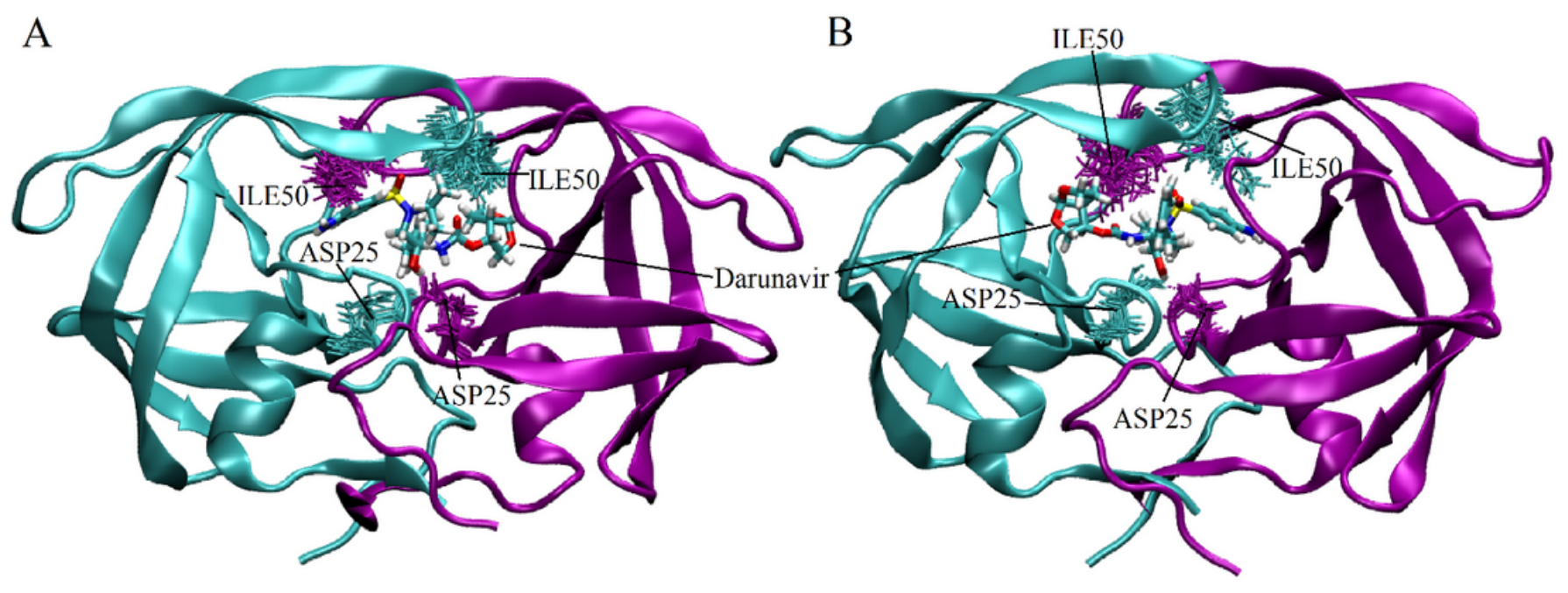

Figure 3

20 superposed Snapshots of ASP25 and lle50 side chains (A:WT-Pr-D and B:MUT-Pr-D) with $10 \mathrm{~ns}$ intervals are illustrated, (cyan: chain-B, purple: chain-A). 
a

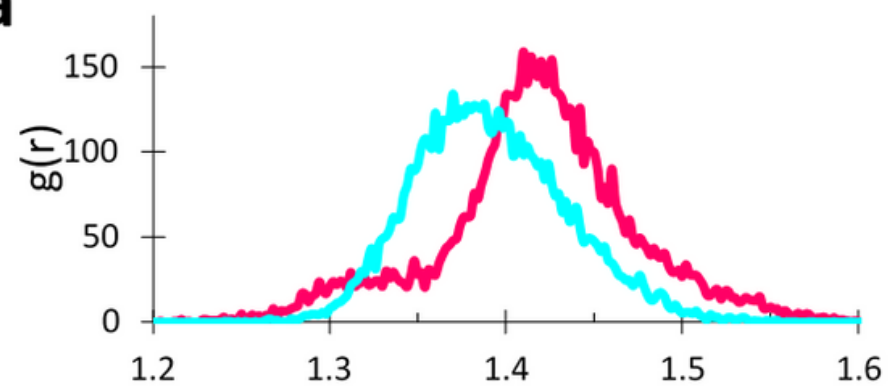

b

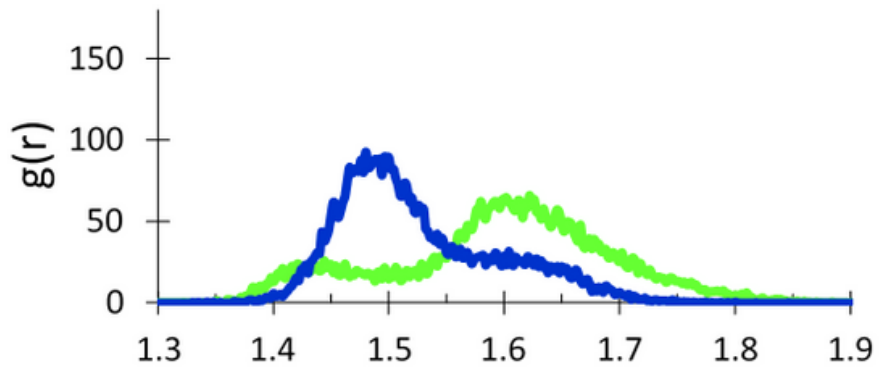

C

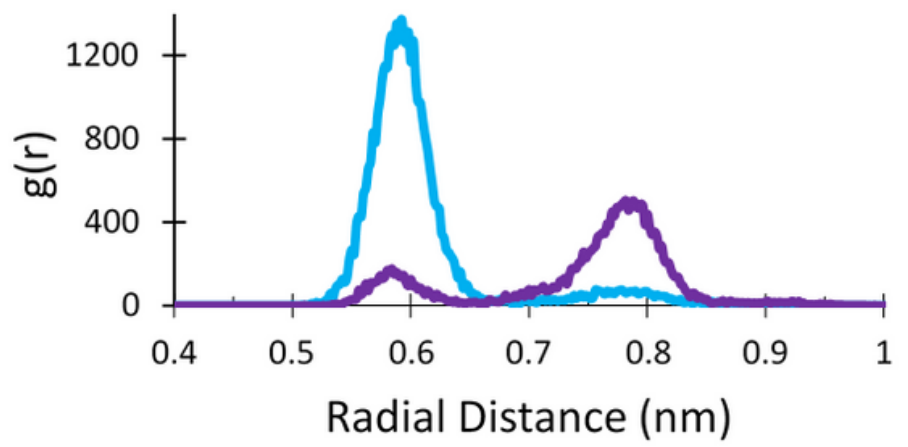

-MUT-Pr-D (Chain A)-ASP25-ILE50

WT-Pr-D(Chain A)-ASP25-ILE50

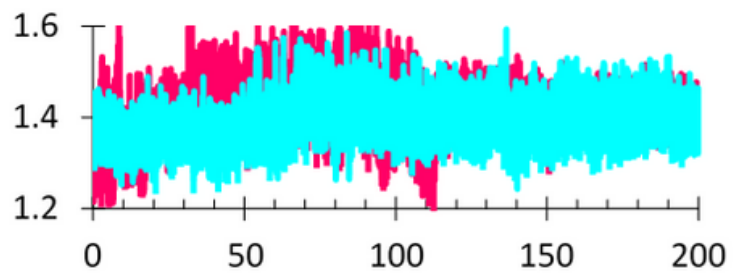

- MUT-Pr-D (Chain B)-ASP25-ILE50 WT-Pr-D(Chain B)-ASP25-ILE50

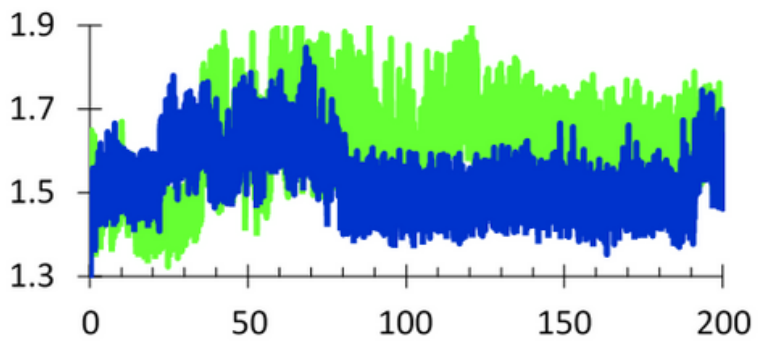

-MUT-Pr-D ILE50-chain A-ILE50Chain B

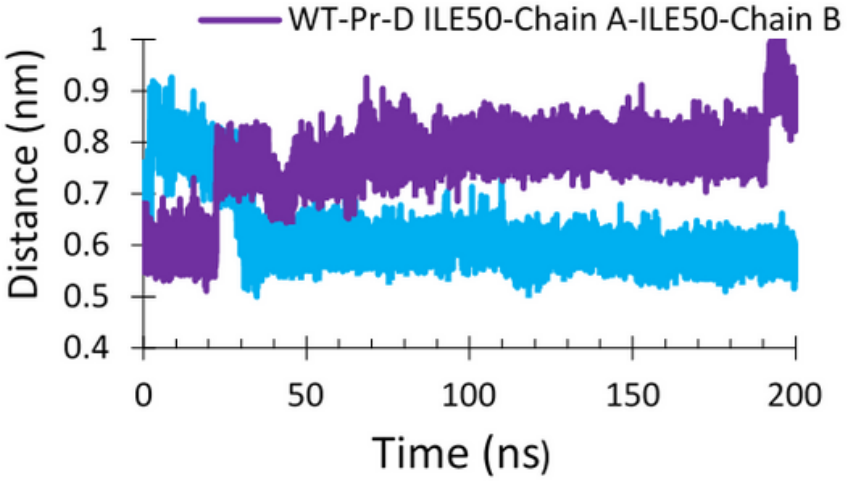

Figure 4

The Ca- $\mathrm{Ca}$ radial distribution functions (RDF) and distances during 200 ns MD simulation for MUT-Pr-D and WT-Pr-D were measured for Asp25-Ile50 in chain-A(a), Asp25-Ile50 in chain-B (b) and lle50 (cain-A)lle50 (chain-B) (c). 




Figure 5

The 20 superposed Snapshots of backbone of flap-tips and residues that are around Darunavir in WT-PrD (A) and MUT-Pr-D (B) with 10 ns intervals. Hydrophobic residues in flap-tips are labeled in picture. 

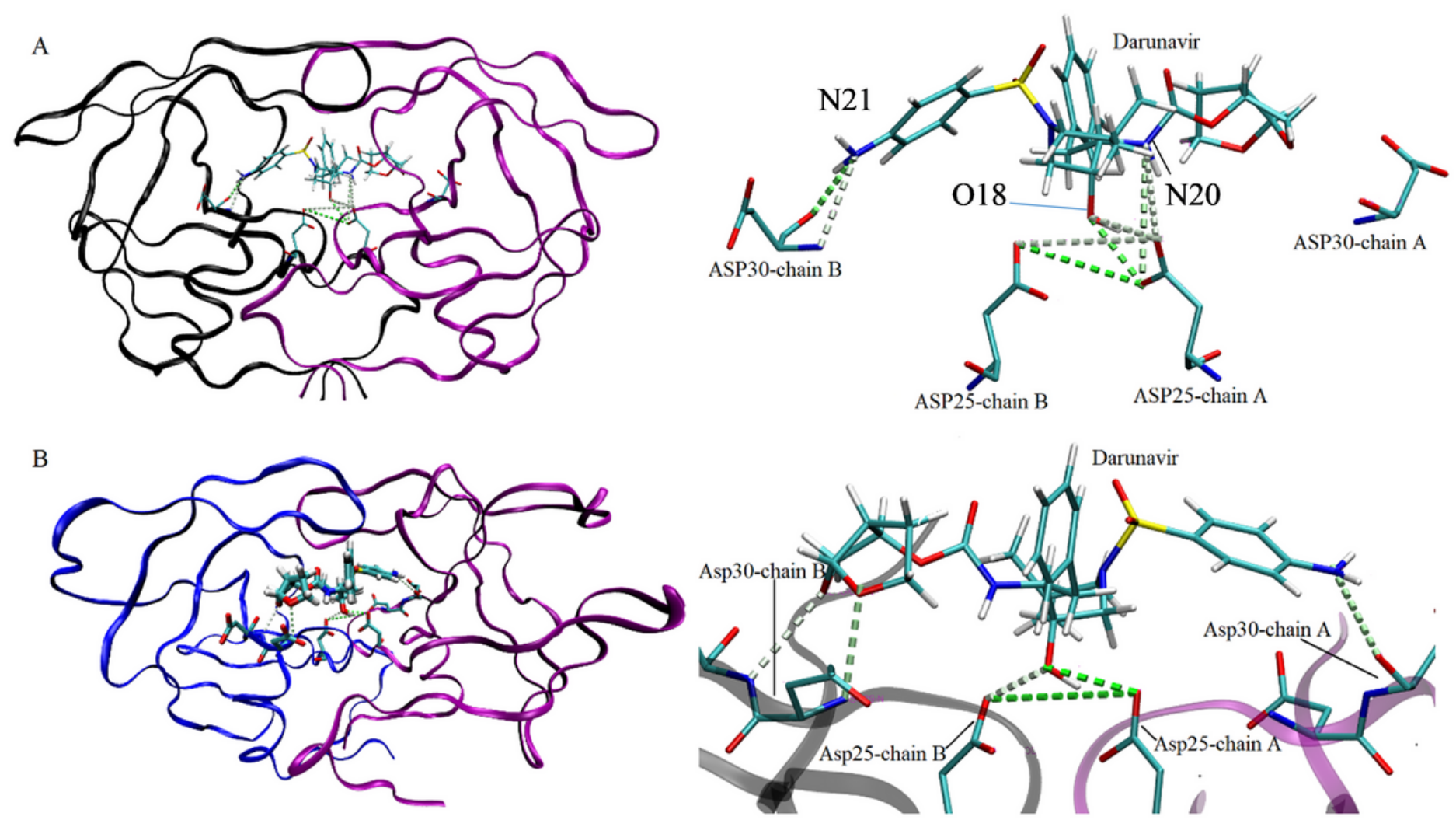

Figure 6

The Pattern of hydrogen bodnds (HB) between Darunavir and WT-Pr (top) and MUT-Pr (bottom). HBs percent in both trajectories are illustrated by color gradient dashed lines. So, that green color dashed lines was used for representing HBs that are formed in all simulation time duration and white color dashed lines representing HBs that are formed at least 10 percent of the simulation duration time. Oxygen atoms are shown by red and nitrogen atoms by blue.

\section{Supplementary Files}

This is a list of supplementary files associated with this preprint. Click to download.

- suplementary.pdf 